\title{
Mapeamento do estado da arte do tema sustentabilidade ambiental direcionado para a tecnologia de informação
}

\author{
Literature review of environmental \\ sustainability related to information \\ technology
}

\author{
Simone SARTORI ${ }^{1}$ \\ Leonardo ENSSLIN ${ }^{1}$ \\ Lucila Maria de Souza CAMPOS' \\ Sandra Rolim ENSSLIN ${ }^{1}$
}

\section{Resumo}

Este artigo tem por objetivo realizar um mapeamento das publicações sobre o tema Avaliação da Sustentabilidade Ambiental para a Tecnologia de Informação e, a partir desse, selecionar um portfólio bibliográfico das publicações mais relevantes e alinhadas segundo a percepção dos autores dessa pesquisa. Para este portfólio, fez-se uma análise bibliométrica visando construir o conhecimento sobre os assuntos mais frequentes: autores, artigos, periódicos e palavras-chave no tema. Caracteriza-se este trabaIho pelo caráter exploratório-descritivo; além disso, apresenta-se abordagens quantitativas e qualitativas, por meio do uso do instrumento de intervenção Knowledge Development Process - Constructivist. Nesse sentido, foram selecionados 25 artigos relevantes e 416 artigos de referências, que passaram a representar o Portfólio Bibliográfico. Na análise bibliométrica dos artigos do Portfólio Bibliográfico e suas referências evidenciam-se: os periódicos "Association for Computing Machinery Computing Surveys"e "Management Information Systems Quarterly"; os artigos "Information Technology and Organizational Performance: An integrative model of Information Technology business value" e "Beyond the Business Case for Corporate Sustainability"; as palavras-chaves "Information Technology" "e"Sustainability"; e os autores mais citados Marie-Claude Boudreau, Tom Butler, Adela Chen, Jason Dedrick, Robert Sroufe, Richard Watson, e Nigel Melville. Esses resultados são singulares devido às delimitações postas pelos autores dessa pesquisa e o processo empregado em sua abordagem é ampla, podendo ser utilizada por outros pesquisadores relacionados a esse tema ou não.

Palavras-chave: Análise bibliométrica. Avaliação de desempenho. Gestão ambiental. Sustentabilidade. Tecnologia de informação.

\section{Abstract}

The aim of this article was to map publications on the issue of Environmental Sustainability Assessment for Information Technology and select a bibliography portfolio of relevant publications according to the perception of authors of this research. For this portfolio the bibliometric analysis aimed at building knowledge on the following most outstanding terms: authors, articles, journals and keywords on the subject. This is an exploratory, descriptive, quantitative, qualitative study using the instrument Knowledge Development Process

\footnotetext{
1 Universidade Federal de Santa Catarina, Programa de Pós-Graduação em Engenharia de Produção. Campus Universitário, Trindade, Caixa Postal 476, 88040-900, Florianópolis, SC, Brasil. Correspondência para/Correspondence to: S. SARTORI. E-mail:<simone.eng.prod@gmail.com>.

Recebido em 13/8/2012, reapresentado em 13/5/2013 e aceito para publicação em 24/6/2013.
} 
from a constructivist perspective. As a result, we selected 25 relevant articles and 416 articles cited in the references, which represent the bibliographic portfolio. The bibliometric analysis of articles in the Bibliographic Portfolio and their references revealed that the most frequent terms were as follows: the journal "Association for Computing Machinery Computing Surveys" and "Management Information Systems Quarterly"; the article "Information technology and organizational performance: an integrative model of Information Technology business value" and "Beyond the Business Case for Corporate Sustainability"; the keywords "Information Technology" and "Sustainability"; and the most cited authors were: Marie-Claude Boudreau, Tom Butler, Adela Chen, Jason Dedrick, Robert Sroufe, Richard Watson, and Nigel Melville. These results are singular due to the delimitations established by the authors of the present study and the process used. However, it is general and it can be used by other researchers in this or other fields.

Keywords: Bibliometric analysis. Performance assessment. Environmental management. Sustainability. Information technology.

\section{Introdução}

Problemas ambientais, como as alterações climáticas, o esgotamento de recursos, a escassez da água e a poluição do ar, entre outros, têm um grande impacto sobre a Terra e seus habitantes; todos esses fatores têm levado a população, em geral, à conscientização dos recursos naturais, para se reverter o processo de degradação ambiental.

A tecnologia é tanto uma das causas desse processo de degradação ambiental, como uma potencial via para amenizar o problema. O uso da tecnologia como em estações de energia movidas a carvão fornece a eletricidade necessária para apoiar um estilo de vida confortável, mas, cria emissões de carbono que contribuem para o aquecimento global. Como alternativa, tecnologias baseadas em energia renovável, como eólica e solar, são as soluções possíveis para a sustentabilidade, embora haja consequências negativas devido aos materiais necessários para construir as turbinas eólicas ou painéis solares (Boudreau et al., 2008).

Entre as tecnologias, destaca-se a Tecnologia de Informação (TI), que, para muitos países, tem sido o principal contribuinte para o crescimento da produtividade no último século, bem como um facilitador de mudanças organizacionais que podem levar a ganhos adicionais de produtividade (Dedrick et al., 2003). Entretanto, o problema ambiental da TI está em suas substâncias tóxicas, na radiação, no consumo de energia e no descarte dos equipamentos (Schmidt et al., 2009). No entanto, os produtos e serviços da TI tornaram-se visíveis em termos de benefícios e custos para a sociedade (Harmon \& Demirkan, 2011).

Até meados da década de 1980, não havia comunidade científica especializada em TI para fins ambientais. Mas durante a última década, a sustentabilidade tem emergido nos estudos e práticas de gestão (Pillmann et al., 2006).

O advento das Tecnologias da Informação vem ocasionando contínuas e profundas transformações nas organizações empresariais e industriais (Nadaes \& Borges, 2008). Essas têm demonstrado comportamentos diferenciados quanto às políticas de gestão, recursos aplicados e instrumentos de controle de gestão socioambiental, configurando problemas, quais sejam: complexos, multicritério, com objetivos conflitantes e incertos. A ciência tem mostrado que para enfrentar tais problemas é recomendado o uso de abordagens prescritivistas e construtivistas em detrimento das abordagens normativistas e descretivistas (Roy, 1993). Muitas são as aplicações de métodos de Avaliação de Desempenho que evidenciam o uso de abordagens construtivistas no referido contexto (Ensslin et al., 2000; Ensslin et al., 2010; Moraes et al., 2010; Tasca et al., 2010; Azevedo et al., 2011; Lacerda et al., 2011; Ram et al., 2011; Ensslin et al., 2012; Rosa et al., 2012). Para essas situações, a avaliação de desempenho tem sido definida como o conhecimento necessário para apreciação de um fato, de uma ideia, de um objetivo ou de um resultado e, também, a base para a tomada de decisão em contextos não bem estruturados, conflituosos e incertos (Ensslin et al., 2010).

Nessas condições, este trabalho tem por objetivo promover um mapeamento das publicações sobre o tema Avaliação da Sustentabilidade Ambiental direcionado para a Tecnologia de Informação. O objetivo divide-se em duas etapas: a) evidenciar um conjunto de artigos (portfólio bibliográfico) com reconhecimento científico, alinhados à visão dos pesquisadores sobre o tema "Sustentabilidade Ambiental em empresas de TI"; e b) estabelecer os mais destacados autores, periódicos e palavras-chave sobre este tema. 


\section{Métodos}

O Instrumento de pesquisa utilizado foi o Knowledge Development Process - Constructivist (ProknowC), proposto por Ensslin et al. (2010) e Ensslin et al. (2012).

O processo Proknow-C é composto de quatro etapas: 1) seleção do portfólio bibliográfico que proporcionará a revisão de literatura; 2) análise bibliométrica do portfólio bibliográfico; 3) análise sistêmica do portfólio bibliográfico; e 4) elaboração dos objetivos de pesquisa.

Nesta pesquisa, sobressaem-se duas etapas iniciais: a seleção do portfólio de artigos sobre o tema de pesquisa e a análise bibliométrica.

\section{Seleção do portfólio bibliográfico}

Para desenvolver um trabalho e construir o conhecimento, o primeiro passo do pesquisador consiste na revisão da literatura acerca do tema (Afonso et al., 2011). O que significa saber buscar, selecionar, avaliar e analisar informações de modo a poder usá-las na construção de conhecimento (Souza, 2011). A seleção do referencial bibliográfico por meio do ProKnow-C realiza-se de forma recursiva por meio das etapas: a) definição dos artigos científicos nas bases de dados; b) estabelecimento de um Banco de Artigos Brutos, seguindo por uma série de procedimentos pré-estabelecidos até a fase de filtragem e seleção do portfólio bibliográfico relevante e alinhado ao tema do pesquisador (Afonso et al., 2011).

\section{Seleção do banco de artigos brutos}

A fim de definir o banco de artigos brutos, parte-se da determinação dos eixos da pesquisa, conforme a percepção do pesquisador. Neste trabalho, tem-se três eixos de pesquisa: a) Gestão Ambiental, que corresponde ao tema central do trabalho; b) Tecnologia de Informação, relacionado a aplicação do primeiro eixo; e c) Avaliação de Desempenho, relacionado aos processos decisórios na busca de aperfeiçoamentos ambientais no tema.

A seleção do Banco de Artigos Bruto é composta por:

a) Definição das palavras-chave: "environmental", "green", "environmental management systems", "ISO 14031" e "sustainability", "Information Technology (IT)",
"Information System (IS)", "performance management", "performance measure", "performance assessment", "performance evaluation" e "performance appraisal".

b) Definição dos bancos de dados: Scopus, Engeneering Village, ISI Web of Knowledge, Science Direct, Wiley e IEEE. Referente ao conteúdo dessas 6 bases de dados, registra-se que foi efetuada a busca pelas 50 combinações de palavras-chave, utilizando os campos título (article title), o resumo (abstract) e as palavras-chave (keywords). A delimitação temporal é de 10 anos (2002 a maio de 2012) e publicações do tipo Journal Article;

c) Levantamento dos artigos nos bancos de dados com as palavras-chave: após conclusão da busca das 12 palavras-chave nas 6 bases de dados, foram selecionados 8506 publicações para compor o portfólio denominado Banco de Artigos Bruto.

\section{Filtragem do banco de artigos brutos}

No processo de filtragem do Banco de Artigos Brutos, analisaram-se as 8506 publicações quanto aos seguintes aspectos: a) artigos repetidos; b) títulos dos artigos alinhados ao tema de pesquisa; c) artigos com reconhecimento científico; d) resumos alinhados ao tema de pesquisa; e e) texto integral dos artigos alinhados com o tema da pesquisa.

Usou-se da biblioteca Endnote para a importação das publicações selecionadas nas bases de dados pesquisadas. Após importação, encontrou-se 586 publicações que não são artigos. Com a eliminação dessas publicações restaram 7920 artigos. Na análise da redundância foram removidos 4662 artigos duplicados, restando 3258 artigos no Banco de Artigos Bruto.

Analisando o alinhamento do título em relação ao tema da pesquisa, foi detectada uma grande quantidade de artigos sobre "Geografic Information Systems (GIS)", fora do escopo da pesquisa. Assim, restaram 289 artigos não duplicados e com título alinhado ao tema de pesquisa, que foram submetidos à análise quanto ao reconhecimento científico no Google Scholar. Para fins dessa análise, entende-se por reconhecimento científico de um artigo como sendo o número de citações encontradas para o artigo após consulta ao Google Scholar. Nesta etapa, os artigos foram divididos em dois grupos: 
a) primeiro grupo com reconhecimento científico; e b) segundo grupo sem reconhecimento científico.

No primeiro grupo, foram selecionados 49 artigos que tiveram ao menos uma citação, sendo esses submetidos à análise do alinhamento do resumo em relação ao tema de pesquisa. Restaram 21 artigos não duplicados, com reconhecimento científico e com título e resumo alinhado ao tema da pesquisa, com 97 autores identificados para uso posterior.

No segundo grupo composto por 240 artigos, encontrou-se 83 artigos publicados há mais de dois anos, mas os autores não tinham artigos selecionados no portfólio do referencial (21 artigos acima colocados). Dando prosseguimento, entre os 240 artigos, verificou-se que 157 artigos foram publicados recentemente (nos anos de 2011 e 2012), possuindo potencial para reconhecimento científico futuro. Esses artigos foram submetidos à análise do alinhamento do resumo em relação ao tema de pesquisa, restando desta análise 9 artigos com resumo alinhado, sendo esses incluídos aos 21 artigos com reconhecimento científico e título/resumo alinhados. Assim sendo, obteve-se 30 artigos não repetidos, com título e resumo alinhados, e com reconhecimento científico.

Em seguida, os 30 artigos selecionados foram analisados em relação ao alinhamento do texto integral com o tema de pesquisa. Dos 30 artigos, 1 artigo não possuía texto completo disponível no Portal de Periódicos da Coordenação de Aperfeiçoamento de Pessoal de Nível Superior (Capes), sendo eliminado. Os demais 29 artigos tiveram seus textos analisados na íntegra e, então, 10 artigos foram excluídos por não estarem alinhados. Os 19 artigos foram considerados constituintes de parte do Portfólio Bibliográfico dos artigos relevantes e alinhados ao tema.

Com o propósito de testar a exaustividade dos 19 artigos do portfólio, esses foram submetidos ao teste de representatividade. Na análise das referências dos 19 artigos, foram constatados 310 artigos, para os quais foram identificadas o número de citações no Google Scholar. Foram encontrados 65 artigos que representam 80\% das citações. Para e esses 65 artigos procedeu-se a leitura integral e se constatou que 6 artigos estavam alinhados, os quais foram incorporados aos 19 artigos do Portfólio Bibliográfico inicial e passaram a constituir o Portfólio Bibliográfico final.
Portanto, o processo de filtragem resultou em um Portfólio Bibliográfico formado por 25 artigos (Anexo).

\section{Resultados}

Apresenta-se a análise das características dos artigos que formaram o Portfólio Bibliográfico alinhado ao tema: avaliação da sustentabilidade ambiental direcionado para a Tecnologia de Informação em termos da seleção dos mais destacados artigos, autores, periódicos e as palavras-chave mais utilizadas no assunto.

\section{Análise bibliométrica}

A análise bibliométrica do Portfólio Bibliográfico consiste no levantamento de estatísticas do conjunto definido de artigos para a gestão da informação e do conhecimento científico do tema de pesquisa (Ensslin et al., 2012). Para tanto, a análise bibliométrica do Portfólio Bibliográfico foi desenvolvida em quatro etapas: a) avaliar o grau de relevância dos periódicos; b) avaliar o reconhecimento científico dos artigos; c) estimar o grau de relevância dos autores; e d) avaliar as palavras-chave mais utilizadas. Para cada uma das etapas acima colocadas, com exceção das palavras-chave mais utilizadas, as análises seguem a seguinte ordem: a) análise bibliométrica dos artigos do Portfólio Bibliográfico; b) análise bibliométrica das referências dos artigos do Portfólio Bibliográfico; c) análise bibliométrica do conjunto formado pelos artigos e suas referências.

Ressalta-se que, para análise bibliométrica, foi composto um conjunto de dados assim distribuídos: 25 artigos que formam o Portfólio Bibliográfico e 416 artigos das referências bibliográficas do tipo Journal Article encontradas nos artigos do Portfólio Bibliográfico.

Estimar o grau de relevância dos periódicos: a primeira análise busca identificar em qual periódico foi publicado o maior número de artigos - dentre aqueles que compõem o Portfólio Bibliográfico. Encontrou-se 18 periódicos diferentes, evidenciando-se entre eles o periódico Management Information Systems Quarterly (MIS Quarterly), com 4 artigos publicados (Figura 1).

A segunda análise evidencia os periódicos com maior número de artigos dentre os citados nas referências 
do Portfólio Bibliográfico. As referências dos artigos do Portfólio Bibliográfico foram publicadas em 178 periódicos diferentes, e destaca-se o periódico MIS Quarterly, com 45 artigos (Figura 2).

A terceira análise compara a relevância dos periódicos dos artigos do Portfólio Bibliográfico e a relevância dos periódicos das referências dos artigos do
Portfólio Bibliográfico, ressaltando-se que: a) o periódico MIS Quartely apresenta destaque no Portfólio e nas referências; b) não há destaque nas referências do Portfólio Bibliográfico; c) não foi encontrado nenhum periódico de destaque no Portfólio Bibliográfico; e d) entre os periódicos presentes nos artigos e nas referências, destacam-se: Business Strategy and the Environment,

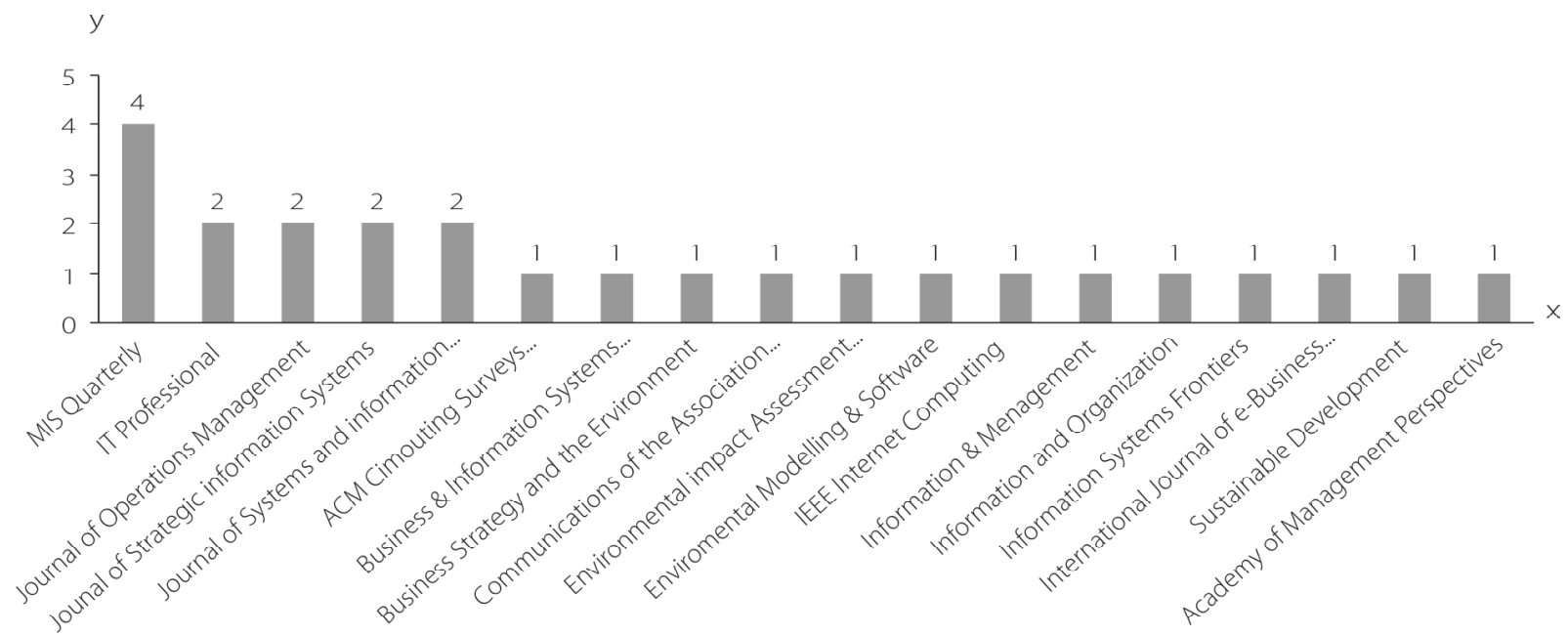

Figura 1. Relevâncias dos periódicos no Portfólio Bibliográfico.

Nota: $x$ : Títulos dos periódicos e y: Números de artigos no Portfólio Bibliográfico. Fonte: Elaborada pelos autores (2012)

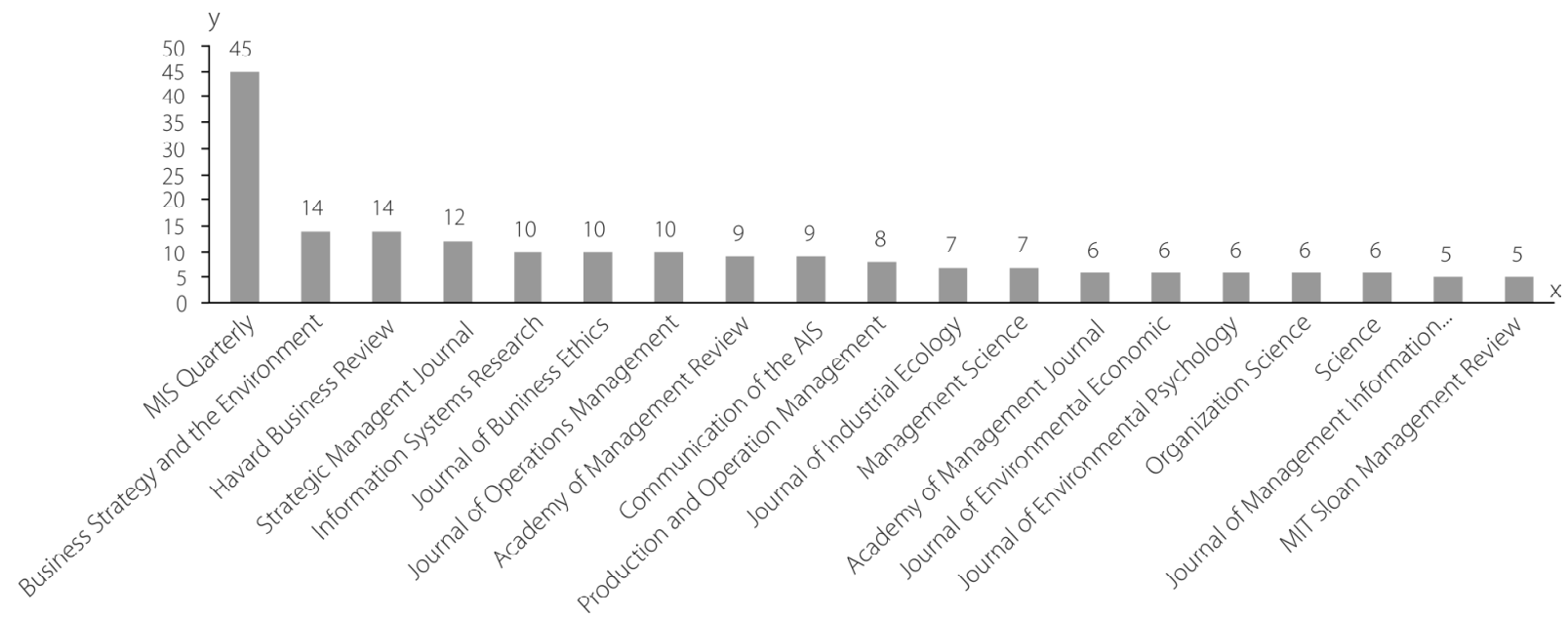

Figura 2. Relevância dos Periódicos nas Referências dos Artigos do Portfólio Bibliográfico.

Nota: x: Títulos dos periódicos e y: Números de artigos nas referências no Portfólio Bibliográfico. Fonte: Elaborada pelos autores (2012). 
Information Technological Professional (IT Professional), Harvard Business Review, Journal of Operations Management, Strategic Management Journal, Journal of Strategic Information Systems, Information System Research e Journal of Systems and Information Technology.

O periódico de destaque, Journal Management Information Systems Quarterly (MIS Quarterly), criado em 1977 e revisado pelo Centro de Pesquisas de Gestão de Sistemas de Informação, Universidade de Gestão Carlson e Universidade de Minnesota, abrange pesquisas nas áreas de Gestão de Sistemas de Informação (SI) e Tecnologia da Informação (TI), Gestão de Recursos de TI, seu uso, impacto, economia e implicações gerenciais, organizacionais, sociais e profissionais (Management Information Systems Quarterly, 2012). Ressalta-se que este é considerado pelos pesquisadores uma das revistas mais prestigiadas na área de Sistemas de Informação.

Estimar o grau de reconhecimento científico dos artigos: o Google Scholar permite consultar a determinação do número de citações para os artigos do Portfólio Bibliográfico, bem como as suas referências, evidenciando o reconhecimento científico.

Na primeira análise, ao avaliar o reconhecimento científico dos artigos do Portfólio Bibliográfico, evidencia-se os artigos: "Information technology and organizational performance", de Nigel Melville, Kenneth Kraemer e Vijay Gurbaxani, com 1153 citações; e, "Beyond the business case for corporate sustainability", de Thomas Dyllick e Kai Hockerts com 635 citações.

A segunda análise objetivou identificar o reconhecimento científico dos artigos do Portfólio Bibliográfico nas referências presentes nos artigos do Portfólio Bibliográfico. Destaca-se, nessa etapa, os artigos "Information systems innovation for environmental sustainability" de Nigel Melville, com 4 citações; os artigos com 2 citações são: "Beyond the business case for corporate sustainability", de Thomas Dyllick e Kai Hockerts e, "An examination of corporate reporting, environmental management practices and firm performance" de Frank Montabon, Robert Soufre e Ram Narasimhan.

A terceira análise compara o número de citações do artigo do Portfólio Bibliográfico e o número de citações obtidas pelo autor mais citado de cada um dos artigos nas referências do Portfólio Bibliográfico, com base no Google Scholar. Verificou-se que: a) nenhum artigo apresenta destaque no Portfólio Bibliográfico; b) um artigo de destaque do Portfólio Bibliográfico foi realizado por Nigel Melville, Kenneth Kraemer e Vijay Gurbaxani, autores de destaque das referências do Portfólio Bibliográfico; e c) o artigo de Thomas Dyllick e Kai Hockerts foi realizado por autores de destaque nas referências do Portfólio Bibliográfico.

Estimar o grau de relevância dos autores: procedeu-se à primeira análise buscando destacar quais os autores de relevância dentre os 50 autores dos artigos do Portfólio Bibliográfico. Ressaltam-se os autores Marie-Claude Boudreau, Tom Butler, Adela Chen, Jason Dedrick, Robert Sroufe, Richard Watson e Nigel Melville com participação em 2 artigos do Portfólio Bibliográfico. Os demais 45 autores possuem participação com apenas 1 artigo no Portfólio Bibliográfico.

A segunda análise busca identificar quais os autores de maior relevância dentre os 684 autores das referências dos artigos do Portfólio Bibliográfico. Encontra-se, portanto, o autor de maior relevância: Richard Watson, com 14 artigos publicados (Figura 3).

A terceira análise evidencia os autores com maior participação no portfólio e suas referências. Portanto, tem-se: a) 7 autores como destaque: Richard Watson, Marie-Claude Boudreau, Adela Chen, Tom Butler, Nigel Melville e Robert Sroufe - cada autor possui 2 artigos no Portfólio Bibliográfico; e b) desses destaca-se Richard Watson, autor de 14 artigos nas referências do Portfólio Bibliográfico, seguido de Marie-Claude Boudreau com 8 artigos e Adela Chen com 7 artigos.

A quarta análise compara o número de artigos realizados pelos autores do Portfólio Bibliográfico e o número de artigos de cada autor nas referências do Portfólio Bibliográfico. Destaca-se (Figura 4): a) seis autores no Portfólio Bibliográfico, quais sejam: Marie-Claude Boudreau, Adela Chen, Tom Butler, Nigel Melville, Jason Dedrick, Robert Sroufe; b) Richard Watson como o autor de destaque no Portfólio Bibliográfico e nas referências do Portfólio Bibliográfico; e, c) não há autores de destaque nas referências do Portfólio Bibliográfico.

Análise do Fator de Impacto dos Periódicos do Portfólio Bibliográfico: o Fator de Impacto, Journal Citation 


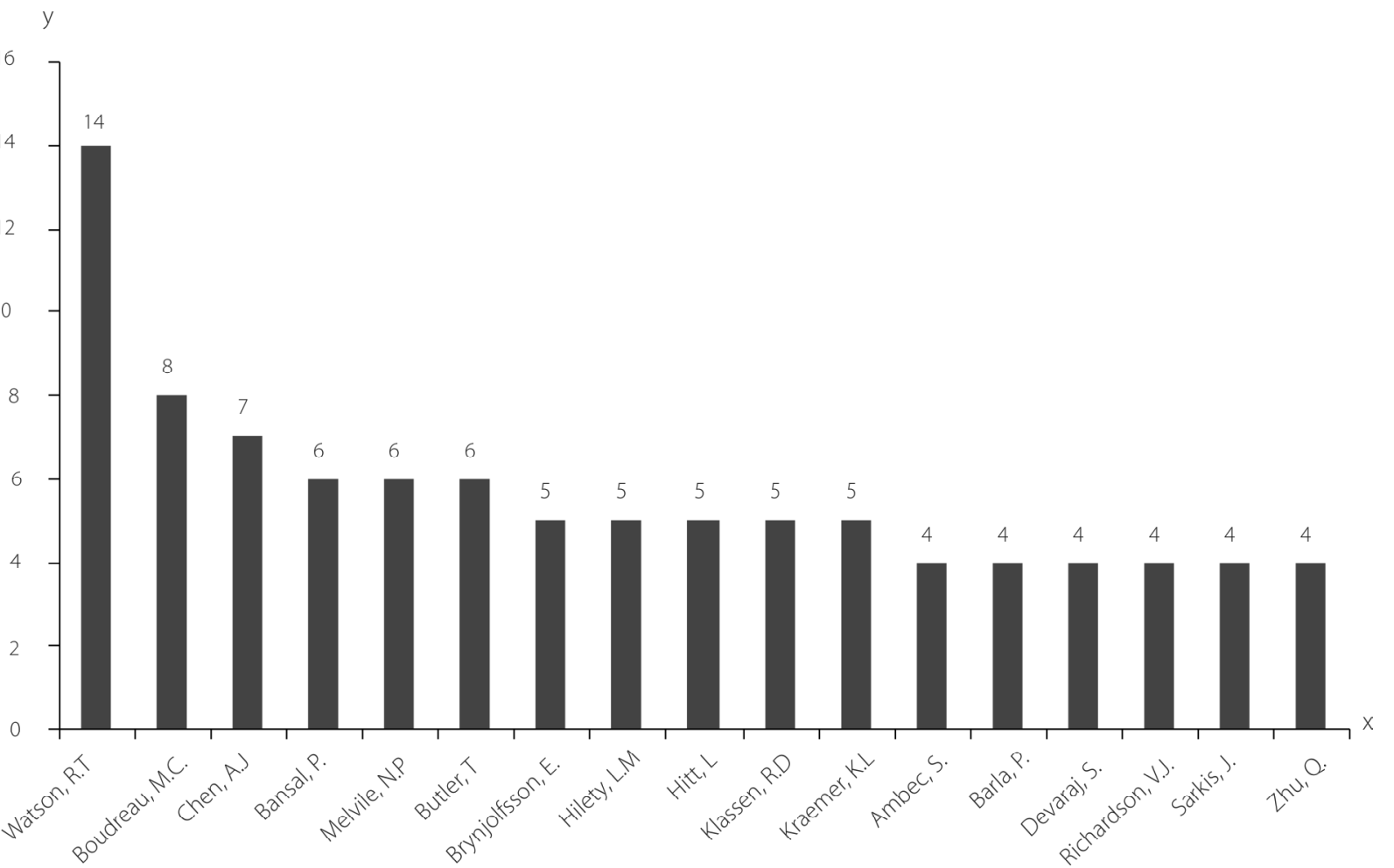

Figura 3. Autores de destaque nas referências do Portfólio Bibliográfico.

Nota: x: Autores das referências do Portfólio Bibliográfico e y: Números de artigos nas referências no Portfólio Bibliográfico. Fonte: Elaborada pelos autores (2012).

Reports (JCR) é uma medida que reflete o número médio de citações de artigos científicos publicados em determinado periódico (Web of Knowledge, 2012). E, o indicador Scientific Journal Ran Kings (SJR), desenvolvido pelo SCImago Journal \& Country Rank, consiste numa medida de visibilidade das revistas científicas (dados a partir de 1996), portanto, mede a "influência científica" média dos artigos de uma revista (SCImago, 2007).

Assim, a primeira análise buscou evidenciar a relevância do assunto dentre os 18 periódicos que compõe o Portfólio Bibliográfico. Destaca-se com maior fator de impacto os periódicos: "Association for Computing Machinery Computing Surveys (ACM Computing Surveys)" com JCR de 4529; "MIS Quartely" com JCR de 4447; e Journal of Operations Management com JCR correspondentes a 4382.

A segunda análise busca evidenciar o indicador SJR nos artigos do Portfólio Bibliográfico, ou seja, a influência científica para o tema desta pesquisa. Destaca-se o ACM Computing Surveys, com maior fator de impacto com SJR de 0.21, seguido dos periódicos Environmental Impact Assessment Review e MIS Quarterly com SJR igual a 0.07; Academy of Management Perspectives e Institute of Electrical and Electronics Engineers Internet Computing (IEEE Internet Computing) com SJR igual à 0.06 .

A terceira análise visa à representatividade do fator de impacto dos periódicos do Portfólio Bibliográfico. Destacam-se: a) o periódico ACM Computing Surveys como destaque em JCR e SJR; b) o periódico MIS Quartely é destaque em JCR; c) não há periódicos de destaque em SJR; d) os periódicos IEEE Internet Computing, Journal of Strategic Information System e Information Systems Frontiers são destaque para o tema da pesquisa.

Estimar as palavras-chave mais utilizadas: esta análise buscou evidenciar quais as palavras-chaves mais 
Autores de destaque do Portfólio Bibliográfico

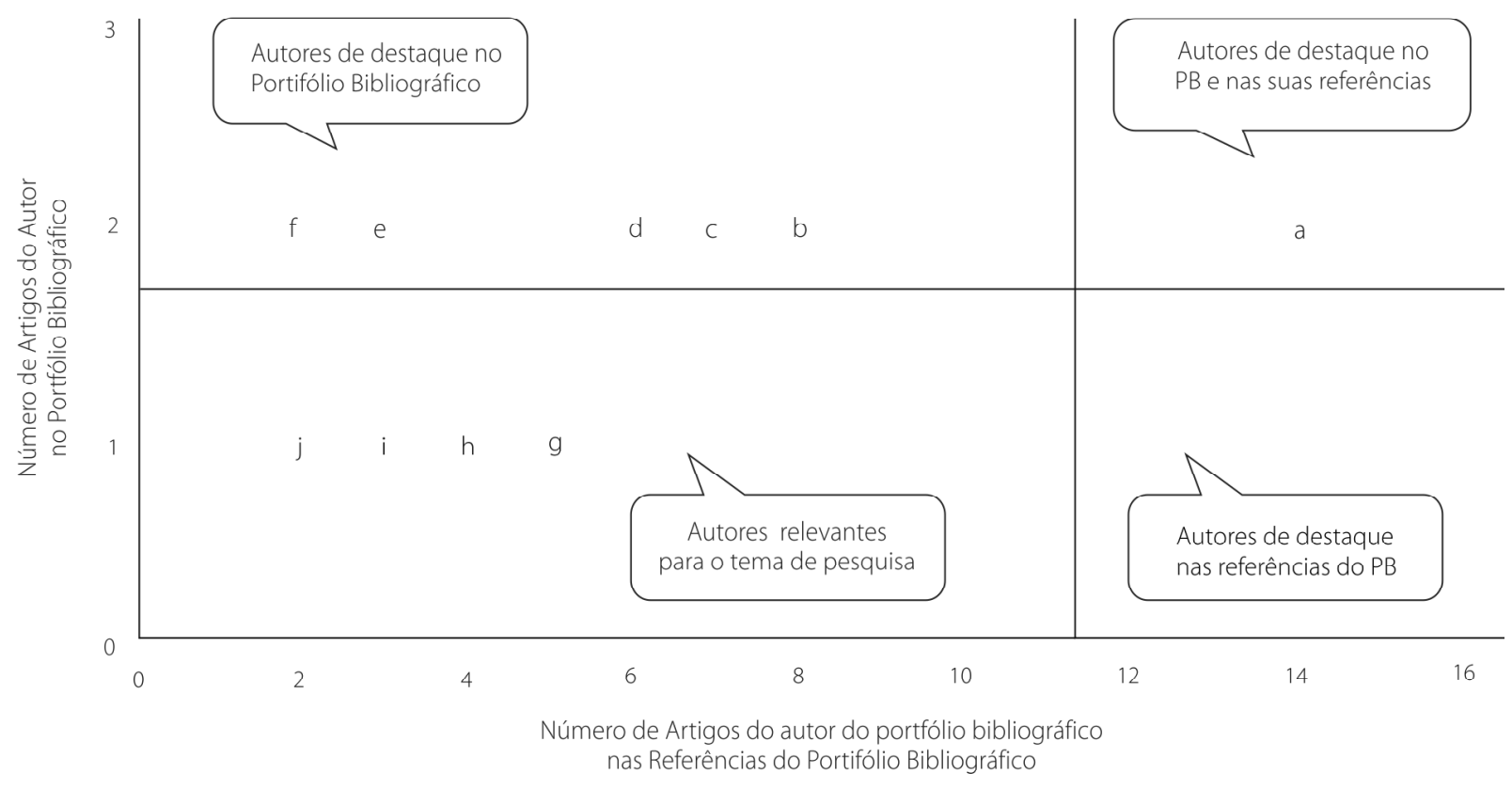

Figura 4. Autores de destaque no Portfólio Bibliográfico.

Fonte: Elaborada pelos autores (2012).

Nota: a) Watson, R. T.; b) Boudreau, M. C.; c) Chen, A.J.W.; d) Butler, T.; d) Melville, N.P.; i) Gurbaxani, V.; i) Lanoie, P.; i) Montabon, F.; i) Murugesan, S.; i) Narasimhan, Re) Dedrick, J.; f) Hilty, L.M.; g) Kraemer, K.L.; h) Ambec, S.; i) Dyllick, T.; j) Annandale, D.; j) Hockerts, K.; j) McGovern, D.

y

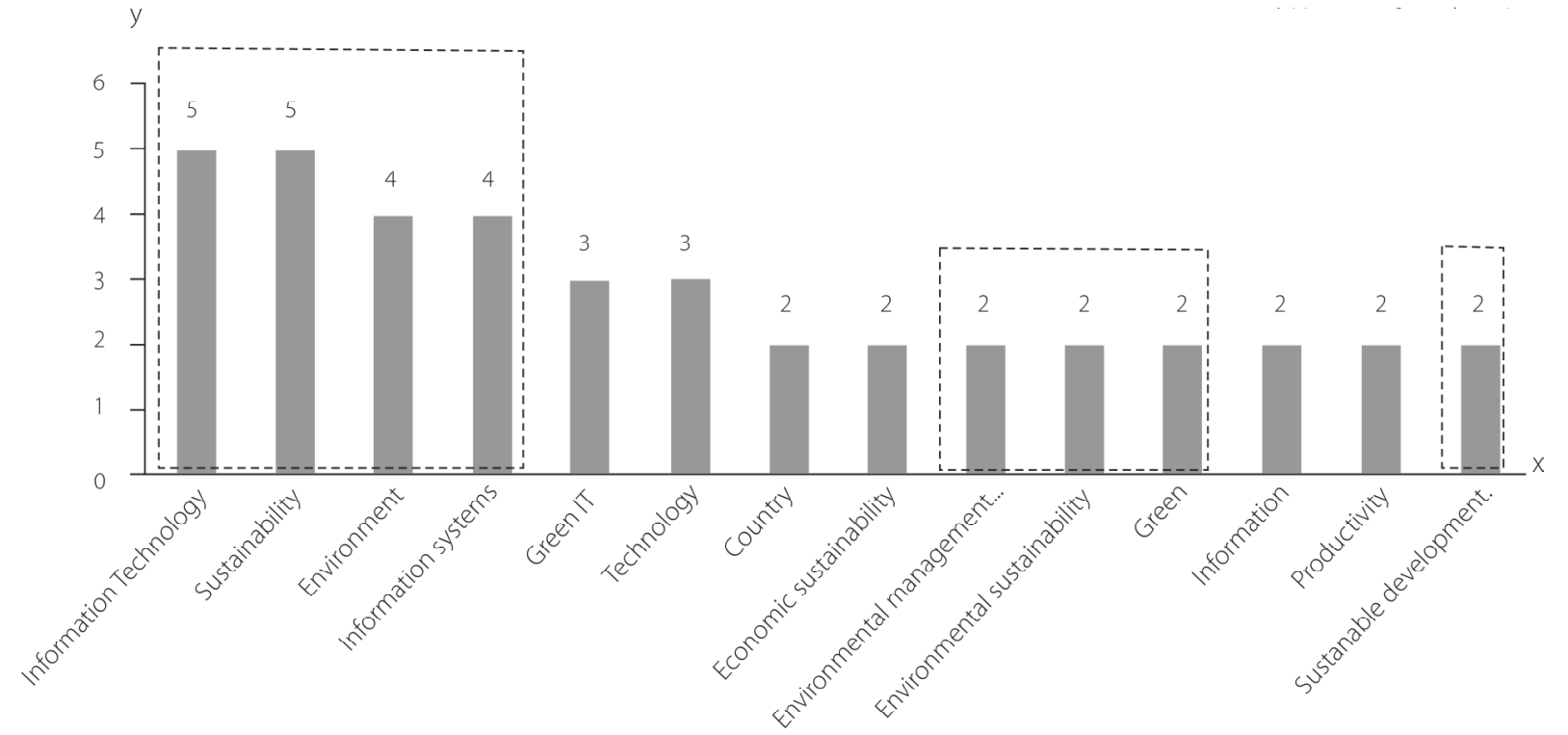

Figura 5. Palavras-chave mais utilizadas no Portfólio Bibliográfico.

Nota: x: Palavras-chave e y: Números de vezes que a palavra-chave aparece nos artigos do Portfólio Bibliográfico. Fonte: Elaborada pelos autores (2012). 
utilizadas nos artigos do Portfólio Bibliográfico. Foram identificadas 90 palavras-chaves utilizadas 116 vezes no Portfólio Bibliográfico. Desse total, 14 foram utilizadas duas vezes ou mais, evidenciando-se que as palavras-chave mais utilizadas foram "information technology" e "sustainability", aparecendo 5 vezes no Portfólio Bibliográfico; as palavras-chave "environment"e "information systems" aparecem 4 vezes (Figura 5). Nessa análise realizou-se a comparação entre as palavras-chave definidas para o tema de pesquisa e aquelas encontradas no Portfólio Bibliográfico.

Destaca-se na Figura 5, em linhas pontilhas e em cor cinza, aquelas palavras definidas para o tema de pesquisa que estavam presentes no Portfólio Bibliográfico. Encontraram-se oito das cinco palavras-chave definidas para o tema da pesquisa, dentre aquelas do Portfólio Bibliográfico utilizadas duas vezes ou mais.

\section{Discussão}

O estudo bibliométrico, inicialmente cunhado como bibliografia estatística (Hulme, 1923), tem por finalidade lançar luz sobre os processos de comunicação escrita em que é apresentada por meio de contagem e análise das várias facetas da comunicação formal.

A bibliometria é uma forma de mapear o atual estado da ciência, fornecendo informações ou indicadores práticos, construindo conhecimento sobre determinado assunto, apresentando um tema em relação à ciência como um todo, um país em relação ao mundo e os pesquisadores individuais em relação às suas próprias comunidades (Pritchard, 1969).

O mais importante na análise bibliométrica é chegar a um conjunto de indicadores consistentes e padronizados (Van Raan, 2005). Com esse intuito, uma primeira indicação para análise bibliométrica num campo específico seria fornecer as características deste campo. Segundo, dar destaque ao papel dos periódicos científicos, e caso os periódicos internacionais forem dominantes ou pelo menos é um meio de comunicação importante, a análise bibliométrica é aplicável. Uma terceira indicação é a análise das citações, pois permite verificar a difusão do conhecimento e a sua utilização numa ponte interdisciplinar. O uso de dados fornecidos pela Internet, "webometrics", é uma oportunidade adicional para auxiliar a análise das citações num mapeamento bibliométrico.

Nesse contexto, o resultado exposto sobre o mapeamento do tema (Avaliação da Sustentabilidade Ambiental direcionado para a TI) propiciou as condições para desenvolver a análise bibliométrica. A integração dos três eixos teóricos - Gestão Ambiental, Tecnologia de Informação e Avaliação de Desempenho -, sustentaram e alinharam a pesquisa de forma exploratória a atender o objetivo geral de construir conhecimento.

Justificando o alinhamento dos resultados apresentados, entende-se que o impacto da TI e o seu papel na sustentabilidade ambiental, sob o título de TI verde, surgiu como um dos principais problemas de gestão da TI (Dedrick, 2010) e só recentemente foram adotadas por pesquisadores da área de SI (Melville, 2010).

Nos últimos anos, os pesquisadores da TI são motivados por um desejo de compreender como e em que medida a sua aplicação leva à melhoria do desempenho organizacional, conformidade ambiental e negócios sustentáveis. Apesar da importância significativa e crescente da TI para avançar na sustentabilidade ambiental e a eficiência do negócio, atualmente existe uma lacuna na literatura dedicada à compreensão do papel da TI no domínio da sustentabilidade ambiental (Bose \& Luo, 2012).

Por sua vez, o tema sustentabilidade, operacionalmente e conceitualmente, é um dos mais complexos que a ciência moderna tem enfrentado (Cabezas \& Fath, 2002). Sob tais circunstâncias, evidencia-se no Portfólio Bibliográfico o comprometimento da TI pelo tema sustentabilidade.

Os resultados apontam para a relevância dos autores e dos periódicos. A saber, a relevância é diferente de fama ou popularidade, mas sim o que é pertinente, se distingue, tem valor e se destaca. Nessas condições, os principais autores que trabalham com esse tema articulam até que ponto e as maneiras pelas quais as atividades atuais são sustentáveis e consequentemente, a viabilidade da sociedade no longo prazo.

Na análise dos periódicos do Portfólio Bibliográfico, 85\% destes periódicos são do campo da Tl e 
são eles que promovem e destacam os debates no tema sustentabilidade. As preocupações ambientais levaram a um significativo aumento no número de periódicos que têm no seu escopo a sustentabilidade. Entretanto, por ser um assunto muito abrangente, os artigos estão dispersos em vários periódicos indicando que o assunto não está consolidado em termos de uma área definida.

Em relação o reconhecimento científico dos artigos, considera-se que quanto mais um trabalho é utilizado como referência para outros, em determinado intervalo de tempo, maior o impacto científico esse trabalho tem. Mas como sabemos se certo número de citações é alto ou baixo? (Van Raan, 2005). O Google Scholar permite consulta para a determinação do número de citações para os artigos, não há um valor (indicador) de referência para comparação.

Ao iniciar uma pesquisa é útil o uso de palavras-chave. As palavras-chave definidas para esta pesquisa e aquelas presentes nos artigos do Portfólio Bibliográfico identificam ideias importantes e servem de referência nas futuras pesquisas sobre o tema. Atenção deve ser dada quando os especialistas num campo específico tendem a citar palavras-chave que são frequentemente utilizadas na sua área e, conjuntamente, em outros contextos; portanto, estas palavras-chave resultam em muitos documentos inadequados, de modo que a amostra não será representativa para o campo considerado (Hinze \& Schmoch, 2004).

De modo geral, as pesquisas sobre o tema procedem de um esforço global, sem fronteiras e em várias áreas. E independente do campo de pesquisa, qualquer um em qualquer lugar pode contribuir para o crescimento do conhecimento e fazer uso coletivo (Arunachalam, 2004).

O mapeamento do estado da arte introduzido neste artigo é uma representação do conhecimento acadêmico em termos quantitativos. Nesse sentido, forneceu-se percepções sobre o tema de pesquisa proposto; assim como, mostrou-se a vantagem existente e baseada no desempenho passado. Isso permitiu dar alguma visão sobre as perspectivas futuras, o que se comprova com a assertiva: os resultados do passado são os melhores preditores para um futuro próximo.
E dentre as principais constatações, cabe destacar que o desenvolvimento de estudos bibliométricos não destitui problemas, pois estes são construções pessoais - abordagem construtivista -, isto é, oportunidades de geração de conhecimento no contexto da avaliação de desempenho personalizada.

\section{Considerações Finais}

A Tecnologia da Informação tem avançado nas últimas décadas, contribuindo para o desenvolvimento de diferenciais competitivos e gerando oportunidades de negócios. Por sua vez, a crescente difusão da TI nos negócios e na vida pessoal chama a atenção para os efeitos ambientais. Na última década, a sustentabilidade emergiu na comunidade científica da TI com estudos e práticas de gestão ambiental. Esse recente impulso pode ser atribuído ao crescimento econômico e às potenciais crises econômicas, à preocupação em torno da disparidade de riqueza e ao esgotamento dos recursos naturais, trazendo à tona a necessidade da compatibilização entre o capital ambiental, o econômico e social.

Nessas condições, este trabalho teve por objetivo geral promover um mapeamento das publicações sobre o tema Avaliação da Sustentabilidade Ambiental direcionado para a Tecnologia de Informação, por meio do uso da ferramenta Proknow-C.

A necessidade de contextualização do conhecimento visa o avanço do progresso científico e o desenvolvimento da ciência. Portanto, o conhecimento aqui construído por meio da bibliometria possibilita percepções iniciais aos leitores interessados no tema sustentabilidade ambiental na TI, por meio da identificação das bases de dados, palavras-chaves, periódicos e autores de destaque e alinhados ao contexto estudado. As publicações não são a única forma de troca de conhecimento, mas certamente possuem elementos e aspectos importantes da ciência.

Importante destacar o estágio de amadurecimento dos eixos: Avaliação de Desempenho, Gestão Ambiental e Tecnologia de Informação. Sozinhos estão consolidados pelas comunidades científica e empresarial, mas juntos são incipientes em termos de publicações científicas. 
Além disso, o uso de um procedimento bibliométrico como o Proknow-C mede o progresso científico, reune conhecimento num campo de muita diversidade e auxilia no processo de tomada de decisão, o que permite explorar, organizar e analisar uma grande massa de dados num contexto restrito, isto é, adaptado ao problema do pesquisador.

Para efeito dessa pesquisa, ressaltam-se as delimitações definidas pelos pesquisadores que influenciaram, de alguma forma, os resultados encontrados: a) as fontes de dados foram restritas ao banco de dados do Portal Capes; b) o período de publicação analisado foi de janeiro de 2002 a maio de 2012; c) os artigos considerados são de cunho prático-empírico; d) o alinhamento do entendimento do conteúdo referente à Gestão Ambiental em Tecnologia de Informação foi realizado segundo a percepção dos pesquisadores.

Como recomendação para futuras pesquisas, sugere-se o uso do Proknow-C para agregar e melhorar as informações e, assim, compreender as decisões envolvidas - para que elas sejam tomadas observandose o que tenha sido prospectado para atingir objetivos relacionados.

\section{Agradecimentos}

Ao Conselho Nacional de Desenvolvimento Científico eTecnológico pelo apoio financeiro.

\section{Referências}

Afonso, M.H.F. et al. Como construir conhecimento sobre o tema de pesquisa? Aplicação do processo Proknow-C na busca de literatura sobre avaliação do desenvolvimento sustentável. Revista de Gestão Social e Ambiental, v.5, n.2, p.47-62, 2011.

Arunachalam, S. Science on the periphery: Bridging the information divide. In: Moed, H.F. et al. (Ed.). Handbook of quantitative science and technology research. Netherlands: Kluwer Academic Publishers, 2004. p.163-183.

Azevedo, R.C.et al. Avaliação de desempenho do processo de orçamento: estudo de caso em uma obra de construção civil. Ambiente Construído, v.11, p.85-104, 2011.

Bose, R.; Luo, X.R. Green IT adoption: A process management approach. International Journal of Accounting and Information Management, v.20, n.1, p.63-77, 2012.

Boudreau, M.C. et al. Green IS: Building sustainable business practices. In: Watson, R.T. (Ed.). Information systems. Athens, GA: Global Text Project, 2008. p.247-261. Available from: <http:// www.ceport.com/files/file/Green\%20IS\%20CePORT\% 20Article.pdf>. Cited: Feb. 23, 2013.

Cabezas, H.; Fath, B.D. Towards a theory of sustainable systems. Fluid Phase Equilibria, v.194-197, n.1, p.3-14, 2002.

Dedrick, J. et al. Information technology and economic performance: A critical review of the empirical evidence. ACM Computing Surveys, v.35, n.1, p.1-28, 2003.

Dedrick, J. Green IS: Concepts and issues for information systems research. Communications of the Association for Information Systems, v.27, n.1, p.11-18, 2010.

Ensslin, L. et al. MCDA: A construtivist approach to the management of human resources at a governmental agency. International Transactions in Operational Research, n.7, p.79-100, 2000.
Ensslin, L. et al. Avaliação do desempenho de empresas terceirizadas com o uso da metodologia multicritério de apoio à decisão-construtivista. Revista Pesquisa Operacional, v.30, n.1, p.125-152, 2010

Ensslin, L. et al. Um estudo sobre segurança em estádios de futebol baseado na análise da literatura internacional. Perspectivas em Ciências da Informação, v. 17, n.2, p.71-91, 2012.

Harmon, R.R.; Demirkan, H. The next wave of sustainable IT. IT Professional, v.13, n.1, p.19-25, 2011.

Hinze, S.; Schmoch, U. Opening the black box: Analytical approaches and their impact on the outcome of statistical patent analyses. In: Moed, H.F. et al. (Ed.). Handbook of quantitative science and technology research. Netherlands: Kluwer Academic Publishers, 2004. p.163-183.

Hulme, E.W. Statistical bibliography in relation to the growth of modern civilization. London: Grafton \& Co., 1923. p.1-72. Available from: <http://ia700401.us.archive.org/33/items/ statisticalbibli00hulmuoft/statisticalbibli00hulmuoft.pdf>. Cited: Feb. 20, 2013.

Lacerda, R.T.O.; Ensslin, L.; Ensslin, S.R. A performance measurement view of IT project management. International Journal of Productivity and Performance Management, v.60, n.2, p.132-151, 2011.

Management Infornation Systems Quarterly. About MIS Quarterly, 2010-2011. 2012. Available from: <http://www. misq.org/about/>. Cited: May 22, 2012.

Melville, N.P. Information systems innovation for environmental sustainability. MIS Quarterly, v.34, n.1, p.1-21, 2010.

Moraes, L. et al. The multicriteria analysis for construction of benchmarkers to support the Clinical Engineering in the Healthcare Technology Management. European Journal of Operational Research, v.200, n.2, p.607-615, 2010. 
Nadaes, A.D.; Borges, M.E.N. Monitoração ambiental no setor de biotecnologia: comportamento de busca e uso de informação em empresas de micro e pequeno portes de Minas Gerais. Transinformação, v.20, n.1, p.99-112, 2008

Pillmann, W.; Geiger, W.; Voigt, K. Survey of environmental informatics in Europe. Environmental Modelling and Software, n.21, p.1519-1527, 2006.

Pritchard, A. Statistical bibliography or bibliometrics? Journal of Documentation, v.25, n.4, p.348-349, 1969.

Ram, C.; Montibeller, G.; Morton, A. Extending the use of scenario planning and MCDA for the evaluation of strategic options. Journal of the Operational Research Society, v.62, n.5, p.817-829, 2011.

Rosa, F.S. et al. Management environmental disclosure: A constructivist case. Management Decision, v.50, n.6, p.1117-1136, 2012.

Roy, B. Decision science or decision-aid science? European Journal of Operational Research, n.66, p184-203, 1993.
Schmidt, N.H. et al. Sustainable information systems management. Business \& Information Systems Engineering, v.1, n.5, p.400-402, 2009.

Scimago. SJR: Scimago journal \& country rank. 2007. Available from: <http://www.scimagojr.com/>. Cited: May 21, 2012.

Souza, E.G. Sociedade da informação e reestruturação produtiva: crítica à dimensão utilitarista do conhecimento. Transinformação, v.23, n.3, p.219-226, 2011.

Tasca, J.E. et al. An approach for selecting a theoretical framework for the evaluation of training programs. Journal of European Industrial Training, v.34, n.7, p.631-655, 2010.

Van Raan, A.F.J. Measurement of central aspects of scientific research: Performance, interdisciplinarity, structure. Measurement, v.3, n.1, p.1-19, 2005.

Web of Knowledge. Journal citation reports: information for new users. 2012. Available from: <http://admin-apps. webofknowledge.com/JCR/help/h_info.htm\#information>. Cited: July 30, 2012. 
Relação do Portfólio Bibliográfico utilizado na pesquisa.

\begin{tabular}{|c|c|c|}
\hline Autores/Ano & Título & Journal \\
\hline Dyllick; Hockerts (2002) & Beyond the business case for corporate sustainability. & Business Strategy and the Environment. \\
\hline Dedrick et al. (2003) & Information technology and economic performance. & $\begin{array}{l}\text { Review of the Empirical Evidence, ACM } \\
\text { Computing Surveys. }\end{array}$ \\
\hline Heo; Han (2003) & $\begin{array}{l}\text { Performance measure of Information Systems (IS) in } \\
\text { evolving computing environments. }\end{array}$ & Information and Management. \\
\hline Melnyk et al. (2003) & $\begin{array}{l}\text { Assessing the impact of environmental management } \\
\text { systems on corporate and environmental performance. }\end{array}$ & Journal of Operations Management. \\
\hline Becker (2004) & Making sustainable development evaluations work. & Sustainable Development. \\
\hline Melville et al. (2004) & $\begin{array}{l}\text { Review: information technology and organizational } \\
\text { performance: an integrative model of IT business value. }\end{array}$ & MIS Quarterly. \\
\hline Pope et al. (2004) & Conceptualizing sustainability assessment. & Environmental Impact Assessment Review. \\
\hline Hilty (2006) & $\begin{array}{l}\text { The relevance of information and communication } \\
\text { technologies for environmental sustainability and } \\
\text { prospective simulation study. }\end{array}$ & Environmental Modelling and Software. \\
\hline Montabon (2007) & $\begin{array}{l}\text { An examination of corporate reporting, environmental } \\
\text { management practices and firm performance. }\end{array}$ & Journal of Operations Management. \\
\hline Standing; Jackson (2007) & An approach to sustainability for information systems. & Journal of Systems and Information Technology. \\
\hline Ambec; Lanoie (2008) & Does it pay to be green? A systematic overview. & The Academy of Management Perspectives. \\
\hline Chen (2008) & Information systems and ecological sustainability. & Journal of Systems and Information Technology. \\
\hline Murugesan (2008) & Harnessing green IT. & IT Professional. \\
\hline Butler; Mcgovern (2009) & $\begin{array}{l}\text { A conceptual model and IS framework for the design and } \\
\text { adoption of environmental compliance management } \\
\text { systems. }\end{array}$ & Information Systems Frontiers. \\
\hline Molla et al. (2009) & An international comparison of green IT diffusion. & International Journal of e-Business Management. \\
\hline Ruth (2009) & Green IT: more than a three percent solution? & IEEE Internet Computing. \\
\hline Schmidt et al. (2009) & Sustainable information systems management. & Business and Information Systems Engineering. \\
\hline Dedrick (2010) & $\begin{array}{l}\text { Green IS: concepts and issues for information systems } \\
\text { research. }\end{array}$ & $\begin{array}{l}\text { Communications of the Association for } \\
\text { Information Systems. }\end{array}$ \\
\hline Melville (2010) & $\begin{array}{l}\text { Information systems innovation for environmental } \\
\text { sustainability. }\end{array}$ & MIS Quarterly. \\
\hline Watson et al. (2010) & $\begin{array}{l}\text { Information systems and environmentally sustainable } \\
\text { development. }\end{array}$ & MIS Quarterly. \\
\hline Butler (2011) & $\begin{array}{l}\text { Compliance with institutional imperatives on } \\
\text { environmental sustainability. }\end{array}$ & Journal of Strategic Information Systems. \\
\hline Dao (2011) & $\begin{array}{l}\text { From green to sustainability: information technology and } \\
\text { an integrated sustainability framework. }\end{array}$ & Journal of Strategic Information Systems. \\
\hline Elliot (2011) & $\begin{array}{l}\text { Transdisciplinary perspectives on environmental } \\
\text { sustainability. }\end{array}$ & MIS Quarterly. \\
\hline Harmon; Demirkan (2011) & The next wave of sustainable IT. & IT Professional. \\
\hline Jenkin et al. (2011) & $\begin{array}{l}\text { An agenda for green information technology and systems } \\
\text { research. }\end{array}$ & Information and Organization. \\
\hline
\end{tabular}



